\title{
Oscillatory Solutions of Third-Order Nonlinear Differential Equations
}

\author{
G.Narendar ${ }^{1}$ and Prof.G.Ramakrishna Rao ${ }^{2}$ \\ ${ }^{1}$ Assistant Professor of Mathematics, Department of Humanities \& Sciences, \\ CVR College of Engineering, Vatunagar, Mangalpalli (V), Ibrahimpatnam (M), \\ R.R.Dist, Telangana State, India. \\ Email: gnriimc@gmail.com \\ ${ }^{2}$ Professor of Mathematics (Retd.) \\ Jawaharlal Nehru Technological University, Kukatpally, Hyderabad-500 072, Telangana State, India. \\ Email: roaprof@yahoo.com
}

\begin{abstract}
In this paper, we are concerned with the oscillation properties of third order nonlinear differential equations of the form

$\left(r(x)\left(r(x) y^{1}(x)\right)^{1}\right)^{1}+q(x) y^{1}(x)+p(x) y^{\lambda}(x)=0, \lambda>0$

Some new sufficient conditions which ensure that every solution oscillates or converges to zero are established. The obtained results extend the result known in the literature for $\lambda=1$. Some examples are considered to illustrate our main results.
\end{abstract}

Keywords and phrases: Oscillatory solution, nonoscillatory solution, Third order, non linear differential equations.

\section{Introduction}

In this section we study the properties of oscillatory solutions of non linear differential equations of third order

$$
\left(r(x)\left(r(x) y^{1}(x)\right)^{1}\right)^{1}+q(x) y^{1}(x)+p(x) y^{\lambda}(x)=0, \lambda>0
$$

Where $r(x), p(x), q(x)$ are continuous functions, with $r(x)>0$ on the interval $I \in(a, \infty),-\infty<a$ and $\lambda$ is positive and is a ratio of two odd integers $\{$ this provides that if $y(x)$ is a solution so does $-y(x)\}$. A nontrivial solution of (1.1) is said to be oscillatory if it has zeros for arbitrarily large values of the independent variable, otherwise it is said to be nonoscillatory. In the linear case with $\lambda=1$ and $r(x)=1$, the properties of oscillatory solutions are discussed in Hanan [01], Lazer [02] and Swanson [03]. The associated nonlinear equations with $r(x)=1$ are the subject discussed by Waltman [04], Heidal [05], Gragus and Venko [06], N.Parhi and S.Parhi [07] have discussed the equation (1.1) with $q(x)=0$ in the

$$
\text { form } y^{111}(x)+p(x) y(x)=0
$$

Also the behavior of solutions of the equations $y^{111}(x)+p(x) y^{11}(x)+q(x) y^{1}(x)+r(x) y^{\lambda}(x)=0$

was considered for oscillation, non oscillation and asymptotic behavior by L.Erbe [08] via a second order equation.
In the discussion of the oscillatory solutions of (1.1) made in the chapter no recourse has been taken to second order equations as well as no change of variables incorporated. The results obtained generalize many theorems mentioned in the above references. To the best of our knowledge nothing is known regarding the qualitative behavior of Equation (1.1) until now.

\section{Main Results}

In this section we present the existence of oscillatory solutions of (1.1) with $p(x)>0, q(x) \geq 0, \lambda$ positive and is a ratio of the odd integers and established that under certain integral conditions any solution of (1.1) which has an initial zero is oscillatory.

2.1 THEOREM: Let $p(x), q(x), r(x)$ are real valued continuous functions on $I=[a, \infty)$ with

$$
\begin{aligned}
& r(x)>0 p(x)>0, q(x) \geq 0, q^{1}(x) \leq 0 \\
& r(x) \in C^{1}(I) \text { and } \operatorname{Lim}_{x \rightarrow \infty}\left|\frac{r^{1}(x)}{r(x)}\right| \text { exists } \\
& A+B \int^{x} \frac{d s}{r(s)}-\int^{x} \frac{d s}{r(s)} \int^{s} p(u) d u d s<0
\end{aligned}
$$

for sufficient large $\mathrm{x}$, where $\mathrm{A}$ and $\mathrm{B}$ are constants and $\lambda$ is positive and quotient of two odd integers, then any containable solution of (1.1) which has zero on I is oscillatory.

PROOF: Let $\mathrm{y}(\mathrm{x})$ be a solution of (1.1) which does not oscillate and let $x_{0}>0$ be the largest zero of $\mathrm{y}(\mathrm{x})$, that is $y(x) \neq 0$ for all $x>x_{0}$. Since $-y(x)$ is also a solution of (1.1), we may assume without loss of generality that there $x_{1}$ exists such that for 
all $x \geq x_{1}, y(x)>0$ and

$y^{1}(x)>0$ for $x \in(x, \alpha)$ for

some

$\alpha$.

$$
\int_{x_{0}}^{x}\left(r\left(r y^{1}\right)^{1}\right)^{1}(s) y(s) d s+\int_{x_{0}}^{x} q(s) y^{1}(s) y(s) d s
$$

Let $x_{2} \in\left(x_{1}, \alpha\right)$. dividing

by $y^{\lambda}$ and integrating from $\mathrm{x}_{2}$ to $\mathrm{x}$, we get

$$
\begin{aligned}
& \int_{x_{2}}^{x} \frac{\left(r\left(r y^{1}\right)^{1}\right)^{1}(s)}{y^{\lambda}(s)} d s+\int_{x_{2}}^{x} \frac{q(s) y^{1}(s)}{y^{\lambda}(s)} d s+\int_{x_{2}}^{x} p(s) d s=0 \\
& \int_{x_{2}}^{x}\left(r\left(r y^{1}\right)^{1}\right)^{1}(s) y^{-\lambda}(s) d s \\
& +\int_{x_{2}}^{x} q(s) y^{1}(s) y^{-\lambda}(s) d s+\int_{x_{2}}^{x} p(s) d s=0
\end{aligned}
$$

Integrating by parts yields

$$
\begin{aligned}
& \frac{r(x) y^{1}(x)}{y^{\lambda}(x)}+\frac{3 \lambda}{2} \int_{x_{2}}^{x} \frac{r\left(y^{1}\right)^{2}(s)}{y^{(\lambda+1)}(s)} d s \\
& +\int_{x_{2}}^{x} \frac{r\left(y^{1}\right)^{2}(s)}{y^{(\lambda+1)}(s)} d s+\frac{\lambda(\lambda+1)}{2} \int_{x_{2}}^{x} \frac{1}{r(s)} \int_{x_{2}}^{x} \frac{r^{2}(u)\left(y^{1}(u)\right)^{3}}{y^{(\lambda+2)}} d u d s \\
& +\int_{x_{2}}^{x} \frac{1}{r(s)} \int_{x_{2}}^{s} \frac{q(u) y^{1}(u)}{y^{\lambda}(u)} d u d s \\
& =\left[\frac{r(x) y^{1}(x)}{y^{\lambda}(x)}\right]\left(x_{2}\right)+k \int_{x_{2}}^{x} \frac{d s}{r(s)}-\int_{x_{2}}^{x} \frac{1}{r(s)} \int_{x_{2}}^{s} p(u) d u d s
\end{aligned}
$$

$<0$, for sufficient large $\mathrm{x}$ where

$$
k=\left[\frac{r(x)\left(r y^{1}\right)^{1}\left(x_{2}\right)}{y^{\gamma}\left(x_{2}\right)}+\frac{\lambda}{2} \frac{r^{2}\left(y^{1}\right)^{2}\left(x_{2}\right)}{y^{(\lambda+1)}\left(x_{2}\right)}\right]
$$

Suppose that $y^{1}(x)>0$ for all $x \geq x_{2}$. Since $y(x)>0$ for all $x \geq x_{0}$, it follows from (2.6) that $y^{1}(x)<0$ for sufficiently large $x$ and this contradict the assumption.

Thus there exists a $x_{3} \geq x_{2}$ such that $y^{1}\left(x_{3}\right)=0$. We Shall now show that $y\left(x_{0}\right)=0$ together with $y^{1}\left(x_{3}\right)=0$ will contradict the positivity of $y(x)$ for all $x>x_{0}$ and hence establish the theorem. Multiplying (1.1) with $y(x)$ and integrating from $x_{0}$ to $x$, we get,

$$
+\int_{x_{0}}^{x} p(s) y^{\lambda+1}(s) d s=0
$$

Let

$$
\begin{aligned}
G[y(x)] & =\frac{1}{2}\left(r^{2} y^{1^{2}}\right)(x) \\
& -\left(r^{2} y y^{11}+r r^{1} y y^{1}\right)(x)-\frac{1}{2} q y^{2}(x)
\end{aligned}
$$

(2.8)

$$
\begin{aligned}
G\left[y\left(x_{0}\right)\right] & =\frac{1}{2}\left(r^{2} y^{1^{2}}\right)(x)-\left(r^{2} y y^{11}+r r^{1} y y^{1}\right)\left(x_{0}\right) \\
& -\frac{1}{2} q y^{2}\left(x_{0}\right)
\end{aligned}
$$

We get

$$
\begin{aligned}
{[y(x)]=} & G\left[y\left(x_{0}\right)\right]-\frac{1}{2} \int_{x_{0}}^{x} q^{1} y^{2} d x \\
& +\int_{x_{0}}^{x} p(s) y^{\lambda+1}(s) d s
\end{aligned}
$$

Since

$y\left(x_{0}\right)=0 ; y^{1}(x)>0 ; G\left[y\left(x_{0}\right)\right]=\frac{r^{2}\left(x_{0}\right)\left(y^{1}\left(x_{0}\right)\right)^{2}}{2} \geq 0$ and

$$
\left.\frac{d\{G[y(x)]\}}{d x}=-\frac{1}{2} q^{1}(x) y^{2}(x)\right)+(x) y^{\lambda+1}(x)>0
$$

We see that $G[y(x)]$ is strictly increasing and vanishes whenever $y(x)$ has a double zero. Using this fact and also since $y\left(x_{3}\right)>0$ and $y^{1}\left(x_{3}\right)=0$ we conclude that 


$$
\begin{aligned}
& G\left[y\left(x_{3}\right)\right]= \\
& \qquad \begin{array}{l}
\frac{1}{2}\left(r^{2} y^{1^{2}}\right)\left(x_{3}\right)-\left(r^{2} y y^{11}+r r^{1} y y^{1}\right)\left(x_{3}\right) \\
\quad-\frac{1}{2} q\left(x_{3}\right) y^{2}\left(x_{3}\right)>0
\end{array}
\end{aligned}
$$

But since $G[y(x)]$ is strictly increasing, it follows from (2.12) that $y^{11}\left(x_{3}\right)<0$. Also we observer that $y(x)$ cannot vanish more than once in $\left[x_{3}, \infty\right)$ for, otherwise suppose that there exists a $x_{4}, x_{4} \in\left(x_{3}, \infty\right) \& x_{4}>x_{3} \mathrm{w}$ $y^{1}\left(x_{3}\right)=0, y\left(x_{4}\right)=0 \& y^{1}(x) \neq 0$ for $x \in\left(x_{3}, x_{4}\right)$.

$$
\begin{gathered}
G\left[y\left(x_{4}\right)\right]=\frac{1}{2}\left(r^{2} y^{1^{2}}\right)\left(x_{4}\right)-\left(r^{2} y y^{11}+r r^{1} y y^{1}\right)\left(x_{4}\right) \\
-\frac{1}{2} q y^{2}\left(x_{4}\right)>0
\end{gathered}
$$

Since $G[y(x)]$ is strictly increasing.

In order that the above inequality may be satisfied we should have $y^{1}\left(x_{4}\right)<0$. but $y^{1}\left(x_{4}\right)=0, y^{11}\left(x_{4}\right)<0$ impl $\mathrm{y}$ that there is a maximum at $x_{4}$ which is a contradiction, since there cannot be a maximum without passing through a minimum. Hence $y^{1}(x)<0$ for $x>x_{3}$ and $\lim _{x \rightarrow \infty} y(x)$ exists.

The rest of the proof is discussed in the following three cases that depend on the sign of $y^{11}(x)$.

CASE (i):- Let $y^{11}(x) \leq 0$ eventually. Then $y(x)$ becomes eventually negative and this is a contradiction.

CASE (ii):- Let $y^{11}(x) \geq 0$. Then since $y^{1}(x)<0$ for $x>x_{3}$ we have $\lim _{x \rightarrow \infty} y(x)$ and

$$
\begin{aligned}
& \frac{G[y(x)]}{r^{2}(x 0} \\
& \leq \lim _{x \rightarrow \infty}\left[\frac{\left(y^{1}(x)\right)^{2}}{2}-\frac{r^{1}(x) y(x) y^{1}(x)}{r(x)}-y(x) y^{11}(x)\right]
\end{aligned}
$$

which is contradiction the fact that $G[y(x)]$ is strictly increasing.

CASE (iii):- If $y^{11}(x)$ changes sign for arbitrarily large $x$ then for $\varepsilon>0$, there exists a sequence $\left\{x_{n}\right\}$, for arbitrarily large values of $x$,for which $-\varepsilon<y^{1}\left(x_{n}\right)<0$, and also another sequence $\left\{s_{n}\right\}, x_{1}<s_{1}<x_{2}<s_{2}<\ldots \ldots \ldots$ of the values if $x$,where $y^{1}(x)$ has relative maxima with $-\varepsilon<y^{1}\left(x_{n}\right)<0$ and $y^{11}\left(s_{n}\right)=0$. Since $\lim _{x \rightarrow \infty} y(x)$ exists and $y^{1}(x)<0, \limsup _{x \rightarrow \infty} y^{1}\left(s_{n}\right)=0$.

Thus we have

$$
\frac{G\left[y\left(s_{n}\right)\right]}{r^{2}\left(s_{n}\right)} \leq \lim _{x \rightarrow \infty}\left[\frac{(\varepsilon)^{2}}{2}-\left|\frac{r^{1}\left(s_{n}\right)}{r\left(s_{n}\right)}\right| y\left(s_{n}\right) \varepsilon\right]
$$

Using (2.1), (2.2) for arbitrarily large values of $S$.

This implies that

$\lim _{n \rightarrow \infty} \frac{G\left[y\left(s_{n}\right)\right]}{r^{2}\left(s_{n}\right)} \leq 0$.

This is again contradiction to the fact that $G[y(x)]$ is strictly increasing.

This completes the proof of the theorem.

\section{Example:}

The equation

$$
y^{111}(x)+y^{1}(x)+2 x^{\frac{-1}{3}}(\operatorname{Sin} x+\operatorname{Cos} x)^{\frac{2}{3}} y^{\frac{1}{3}}=0
$$

satisfies the conditions of the theorem (2.1).Hence the solutions are oscillatory. In fact $y(x)=x(\operatorname{Sin} x+\operatorname{Cos} x)$ is one of the solutions, which is oscillatory.

\section{Conclusion}

The requirement that the solutions (1.1) must have an initial zero on $\mathrm{I}$ is essential to obtain the conclusion. With $r(x) \equiv 1, q(x) \equiv x, p(x) \equiv 1$ and $\lambda=1$ on $[0, \infty)$, the equation (1.1) becomes

$y^{111}(x)+x y^{1}(x)+y=0$

The conditions (2.1), (2.2) are satisfied. The condition (2.3) is verified as follows.

$$
A+B \int_{t_{0}}^{t} \frac{d s}{r(s)}-\int_{t_{0}}^{t} \frac{1}{r(s)} \int_{t_{0}}^{s} p(u) d u d s=A+B \int_{t_{0}}^{t} d s-\int_{t_{0}}^{t} \int_{t_{0}}^{s} d u d s
$$




$$
=A+B\left(t-t_{0}\right)-\frac{\left(t-t_{0}\right)^{2}}{2} \rightarrow-\infty \text { as } t \rightarrow \infty .
$$

But the equation (2.18) is a $C_{1}$ equation and all $C_{1}$ equations have non-oscillatory solutions.

\section{ReFERENCES}

[01] M.Hanan, "Oscillation criteria for third order linear differential equations," Pacific J.Math; vol.11, pp. 919 944, 1961.

[02] A. C. Lazer, "The behaviour of solutions of the differential equation $y^{111}+p y^{1}+q y=0$," Pacific Journal Math., vol.17, pp.435 - 466, 1966.

[03] C.A.Swanson, Comparision and oscillation theory of linear differential equation," Academic press, Newyork, 1968

[04] Paul Waltman, "Oscillation criteria for third order nonlinear differential equations," Pacific Journal of Mathematics, vol.18, pp.384-389, 1966.

[05] J. W. Heidel. and Don B. Hinton, "The existence of oscillatory solutions for a nonlienar differential equation," SIAM J.Math. Anal. Vol 3, No.2, pp.344-351, 1972.

[06] M.Gregus and J.Vencko, on oscillatory properties of solutions of certain nonlinear third order differential equation," czechoslovak Mathematical Journal,vol.42 (117), pp.675-684, 1992.

[07] N.Parhi and S.Parhi, "Oscillation and nonoscillation theorems for nonhomogenous third order differential equations,"Bull.Inst. of Math. Academia Sinica, vol.11, pp.125139, 1983.

[08] Erbe, "Oscillation, Nonoscillation and Asymptotic behaviour of third order Nonlinear Differential equations," Anali di Mate puraed Appl. Vol. CX, pp.373-393, (1976)

[09] J. Anders, "Boundedness of solutions of the third order differential equation with oscillatory restoring and forcing terms," Czechoslovak Mathematical Journal, vol.36 (III), pp.1-6, 1986.

[10] J.H.Barrett, "Oscillation theory of ordinary linear differential equation," Advances in Maths. , vol.3, pp. 415-509, 1969

[11 ] N. Pahri and P. Das, "Asymptotic property of solutions of a class of third-order differential equations," proc. Amer. Math. Soc. Vol.110, pp.387-393, 1990.

[12] V.S.H.Rao and R.S.Dahiya, " properties of solutions a class of third order linear differential equations,"periodica Mathematica Hungarica 20, (1989)', 177-184.

[13] L.H.Erbe and V.S.H.Rao, "Nonoscillation Results for third order Nonlinear Differential equations, JMAA, vol.125, pp.471-482, (1987).

[14] L.Erbe and V.Sreehari Rao, "Nonoscillation critria for forced second order Nonlinear Equations," Journal of Mathematical Analysis and, Applications, Vol. 97, pp.202-213, Nov.1983.

[15] L.Erbe and V.Sreehari Rao, "On the generalized EmdnFowler and Thomas-Ferni Differential equations," Bull. Alld. Math.Seo Vol. 5, pp. 21-78, 1990.

[16] L.Erbe, V.Sreehari Rao and K.V.V.Sehsagiri Rao, "Non- oscillation and asymptotic properties for a class of forced second order nonlinear equations, Math. Proc. camb. Phil.Soc. vol.95, pp.155-163, 1984. 